\title{
Determination of the dispersion of low frequency waves downstream of a quasiperpendicular collisionless shock
}

\author{
M. A. Balikhin ${ }^{1}$, L. J. C. Woolliscroft ${ }^{1}$, H. St. C. Alleyne $^{2}$, M. Dunlop ${ }^{3}$, M. A. Gedalin ${ }^{4}$ \\ ${ }^{1}$ Department of Automatic Control and Systems Engineering, The University of Sheffield, Sheffield, UK \\ 2 Department of Physics, The University of Sheffield, Sheffield, UK \\ ${ }^{3}$ Space Physics Group, Imperial College of Science and Technology, London, UK \\ ${ }^{4}$ Physics Department, Ben-Gurion University, Beer-Sheva, Israel
}

Received: 13 May 1995 / Revised: 17 July 1996 / Accepted: 30 July 1996

\begin{abstract}
A method of wave mode determination, which was announced in Balikhin and Gedalin, is applied to AMPTE UKS and AMPTE IRM magnetic field measurements downstream of supercritical quasiperpendicular shock. The method is based on the fact that the relation between phase difference of the waves measured by two satellites, Doppler shift equation, the direction of the wave propagation are enough to obtain the dispersion equation of the observed waves. It is shown that the low frequency turbulence mainly consists of waves observed below $1 \mathrm{~Hz}$ with a linear dependence between the absolute value of wave vector $|\boldsymbol{k}|$ and the plasma frame wave frequency. The phase velocity of these waves is close to the phase velocity of intermediate waves $V_{\text {int }}=V_{a} \cos (\theta)$.
\end{abstract}

\section{Introduction}

Waves play a crucial role in the many processes which take place in space plasmas, so the study of wave turbulence is an important part of experimental space plasma investigations. The main task of plasma wave experiments is the determination of the energy stored in turbulence and the distribution of this energy among excited wave modes. Estimation of the amount of energy stored in the waves is a relatively easy task, which can be done directly from measurements of wave amplitudes. However, the determination of plasma modes of observed waves is a more complicated problem which is related to the problem of distinguishing between spatial and temporal variations. The primary characteristic of a plasma mode is the dispersion relation between the wave vector and the frequency. This cannot be measured directly. For this reason only the secondary characteristics of the mode such as observed frequency,

Correspondence to: M. Balikhin ratio of the amplitudes of the various physical parameters of the wave (e.g. electric field to magnetic field, or amplitude of magnetic field oscillations to the magnitude of electron density fluctuations), or wave polarization and propagation properties, were often used for the mode identification (e.g. Vaisberg et al., 1983; Rodriguez and Gurnett, 1975; Lacombe et al., 1990). These experimentally obtained secondary characteristics were compared with theoretically estimated equivalent quantities to determine the mode of the waves. These quantitative values of the characteristics often are very sensitive to the fine features of the plasma state and at the same time they are usually derived analytically under very specific conditions such as, for example, an exactly Maxwellian plasma distribution, or infinitively small wave amplitude. It is hard to accept this quantitative comparison of wave characteristics with the real finite amplitude waves observed in space plasmas. By contrast the general behavior of the dispersion relation is not so sensitive and can more often point unambiguously to the observed mode. Since the first two point measurements of wave fields became available, the experimental determination of the dispersion relation have been possible. In the present study a method for the determination of the dispersion relation is based on the calculation of the complex coherency function of two data sets. The phase of the coherence function $\phi(v)$ at the given frequency $(v)$ can be related to the projection of the wave vector $K_{S}(v)$ on the separation direction of the satellites. The direction of the wave vector can be determined by standard methods (e.g., minimum variance). The wave vector itself can be calculated on the basis of its direction and the projection on the separation line. The dispersion relation can then be calculated in the plasma rest frame making use of the Doppler shift equation, providing knowledge of the plasma bulk velocity and wave vectors for each frequency. This method is tested on magnetic field data obtained from two satellites, AMPTE UKS and AMPTE IRM, downstream of a supercritical quasiperpendicular bow shock. 


\section{Coherency function of two data sets}

In this section we consider some two dimensional, real, random, stationary process $X_{t}, Y_{t}$. Under stationarity we mean second order stationarity i.e., that all first and second moments of the process do not depend on time. The spectral density $S_{X_{t}}(v)$ of a stationary random discrete process $X_{t}$ can be estimated from the $N$ point time series of the process as:

$S_{X_{t}}(v)=T \sum_{k=0}^{N-1} X_{t}(k) \exp (-j 2 \pi v k T)$,

where $j=\sqrt{-1}$ and $T$ is the sampling period.

The coherency function $\gamma_{X Y}(v)$ and the phase $\phi_{X Y}(v)$ of such stationary processes are defined by the following equations:

$\gamma_{X Y}^{2}(v)=\frac{\left|C_{X Y}(v)\right|^{2}}{\left|C_{X X}(v)\right|\left|C_{Y Y}(v)\right|}$

$\phi_{X Y}(v)=\arctan \left(\frac{\operatorname{Im} C_{X Y}(v)}{\operatorname{Re} C_{X Y}(v)}\right)$,

where:

$C_{X Y}(v)=\left\langle S_{X_{t}}^{*}(v) S_{Y_{t}}(v)\right\rangle$.

$\langle\ldots\rangle$ means averaging.

For $\gamma_{X Y}(v)$ the following condition must be valid (e.g., Grenander and Rosenblatt, 1957):

$0 \leq \gamma_{X Y}(v) \leq 1$.

The physical meaning of the coherency function is similar to the meaning of the correlation function but only for Fourier components at some frequency $v$. If the value of the coherency function $\gamma_{X Y}(v)$ is close to 1 that means that Fourier components at the frequency $v$ of two processes $X_{t}$ and $Y_{t}$ are strongly related. If $\gamma_{X Y}(v)$ is small these components are statistically independent of each other. The phase $\phi_{X Y}(v)$ seen from Eq. (2) is simply a phase difference between two Fourier components at a frequency $v$. If the coherency is low the significance of the phase is also low: if the coherency is zero then the values for the phase are distributed randomly between $0^{\circ}$ and $360^{\circ}$. The variation of the phase is much less if the value of the coherence is high. The variation of the phase can be used as an indicator of the value of the coherency.

It is necessary to mention one property of the phase function (e.g., Otnes and Enochson, 1978). Suppose $x_{t}(t)$ is a time-delayed version of $y_{t}(t)$ :

$x_{t}(t)=y_{t}(t-\tau)$

The Fourier transform of $x_{t}(t)$ is:

$$
\begin{aligned}
X_{f}(v) & =\int_{-\infty}^{\infty} x_{t}(t) \exp (-j 2 \pi v t) \mathrm{d} t \\
& =\int_{-\infty}^{\infty} y_{t}(t-\tau) \exp (-j 2 \pi v t) \mathrm{d} t \\
& =\exp (-j 2 \pi v \tau) \int_{-\infty}^{\infty} y_{t}(t) \mathrm{d} t=\exp (-j 2 \pi v \tau) Y_{f}(v) .
\end{aligned}
$$

The phase in this case is defined by following equation:

$\phi_{X Y}(v)=-2 \pi v \tau$,

so the phase $\phi_{X Y}(v)$ is a straight line with the slope $-2 \pi \tau$.

\section{Techniques to determine the dispersion relation}

Suppose that in the plasma rest reference frame a wave can be described as:

$\boldsymbol{B}(\boldsymbol{r}, t)=\sum_{\omega_{p . f}} \boldsymbol{B}\left(\omega_{p . f .}\right) \exp j\left(\boldsymbol{k} \cdot \boldsymbol{r}-\omega_{p . f .} t\right)+$ C.C.,

where C.C. is the complex conjugate term. Frequency $\omega_{p . f}$. and wave vector $\boldsymbol{k}$ are related by the dispersion relation:

$\boldsymbol{k}=\boldsymbol{k}\left(\omega_{p . f .}\right)$.

If the two point measurements are carried out with a time shift $\Delta t$ and a separation vector $\boldsymbol{R}$ the phase shift of two measured time series can be estimated as:

$\Delta \psi=\boldsymbol{k} \cdot \boldsymbol{R}-\omega_{\text {p.f. }} \Delta t=|k||R| \cos (\vartheta)-\omega_{\text {p.f. }} \Delta t$,

where $\vartheta$ is the angle between the wave vector and the separation vector. Plasma frame frequency $\omega_{p . f}$. is not a directly measured quantity. The waves are detected in the satellite frame at frequency $\omega_{1}$, which is shifted from the plasma reference frame frequency due to the Doppler effect:

$\omega_{1}=\omega_{p . f .}+\boldsymbol{k} \cdot \boldsymbol{V}_{0}$.

So the phase shift can be rewritten as:

$\Delta \psi=|k| \cdot|R| \cos (\vartheta)-\left(\omega_{1}-|k| \cdot\left|V_{0}\right| \cos (\varphi)\right) \Delta t$,

where $\varphi$ is the angle between the wave vector and the plasma bulk velocity direction.

If the calculation of the phase shift according to Eq. (2) provides some dependence of phase upon the measured frequency:

$\Delta \psi=\Psi\left(\omega_{1}\right)$

the magnitude of wave vector can be determined as:

$|k|\left(\omega_{1}\right)=\frac{\Psi\left(\omega_{1}\right)+\omega_{1} \Delta t}{|R| \cos (\vartheta)+\left|V_{0}\right| \Delta t \cos (\varphi)}$.

The absolute value and the direction of plasma bulk velocity $\boldsymbol{V}_{0}$ usually can be determined from ion-plasma experimental data. The direction of the wave vector, which is needed for the determination of $\vartheta$ and $\varphi$, can be calculated for magnetic field measurements by the standard methods of the minimum variance or maximum variance (Sonnerup and Cahill 1967; Lacombe et al., 1992). Then the dispersion relation (5) can be calculated from Eqs. $(6,7)$ if the direction of the wave vector is known.

This method has the obvious confidence parameters: the value of the coherency function and the ratios of the eigenvalues of the variation matrix (Sonnerup and Cahill, 1967). Only the high level of the coherency function makes the values of the phase meaningful. The 
existence of the confidence parameters makes simple the possible onboard real-time realisation of this method.

The fact that three equations [the equation for the determination of the wave vector (7); the equation for the Doppler effect (6); the equation for the determination of the direction of wave propagation] are enough to determine the dispersion relation was first noted in (Balikhin and Gedalin, 1993). Instead of the coherency function for the relation between phase shift and the wave vector projection, another approach can be used. Making use of the concept of the local wave number (Beall et al., 1982) a two-dimensional histogram of the projection of wave vectors on the direction of $\boldsymbol{R}$ can be produced. Then the best fit procedure can be used to extract, from this two-dimensional histogram, a curve which corresponds to the relation between the projection of the wave vector and the observed frequency. The other two equations together with this relation can be used to determine the wave dispersion. This method does not have obvious confidence parameters. This is particularly due to the necessity of extracting a one-dimensional curve from a two-dimensional histogram. It therefore, for example, cannot be so readily implemented in real-time onboard processing. Recently the Beall et al., (1982) method was applied to the study of low frequency turbulence in a quasiparallel shock (Dudok de Wit et al., 1995).

\section{Experimental data}

Some data obtained from AMPTE UKS and AMPTE IRM magnetometers are analyzed in the present study. The magnetic field experiments on AMPTE UKS and AMPTE IRM are described in Lühr et al., (1985) and Southwood et al., (1985). Both data sets have the same sampling frequency $8 \mathrm{~Hz}$. (The proposed method can be used also when sampling rates are different, but in that case interpolation of one of the signals must be made.) The time of AMPTE UKS data sets corresponds to the moment of the measurements; the time of AMPTE IRM data corresponds to the moment when these data were received on the Earth. To determine the correspondence between the data sets we need to follow the procedure described in Schwartz et al., (1992) and to subtract from the AMPTE IRM time delay $t_{d}$. The value $t_{d}$ is the time which is needed for the signal to propagate from the AMPTE IRM satellite to the Earth's surface:

$t_{d}=\frac{L_{i r m}}{c}$

$L_{i r m}$ is the distance from the Earth's surface to the AMPTE IRM at the moment of the measurement. For the considered time period $L_{\text {irm }} \approx 79000 \mathrm{~km}$ so $t_{d} \approx 0.264 \mathrm{~s}$. The first point which was used in the AMPTE UKS data set corresponds to the time 14:00:00:076 AMPTE UKS time, the first point of AMPTE IRM data sets relates to the 14:00:00:375 AMPTE IRM time. So (fortunately) the difference in the absolute time between two data sets is $0.035 \mathrm{~s}$, this time shift corresponds to a frequency higher than the Nyquist frequency. Variations at such frequencies are strongly attenuated in both magnetometers (Lühr et al., 1985; Southwood et al., 1985), so we can consider the measurements as simultaneous.

The ion plasma parameters (density, velocity etc.) used in the present study, were kindly provided by A.J. Coates and S.J. Schwartz on the basis of the AMPTE UKS three-dimensional ion experiment (Mullard Space Science Laboratory) data. Upstream parameters were calculated on the basis of solar wind mode data (Coates et al., 1985). Downstream parameters were calculated on the basis of fast time resolution mode data.

\section{Bow shock crossing on 20 October 1984 at about 14:10 UT}

We consider the Earth's bow shock which was measured by AMPTE UKS and IRM at about 14:10 UT on 20 October 1984. The position of AMPTE UKS at 14:10 UT was $\left(12.19 R_{e} 0.77 R_{e}-0.40 R_{e}\right)$ (GSE). Three components and the absolute values of the magnetic fields measured by AMPTE UKS and AMPTE IRM are shown in Figs. 1 and 2. The time scale on these figures is in seconds after 14:00:00 UT. In the downstream region, waves which were observed from $650 \mathrm{~s}$ to $1100 \mathrm{~s}$ are studied. The upstream bulk velocity $V_{0}$ was $680 \mathrm{~km} \cdot \mathrm{s}^{-1}$. The angle between the upstream magnetic field and the normal to shock front, estimated on the basis of the coplanarity theorem was $47^{\circ}$. The upstream region ion inertial length, $c / \omega_{p i}$, for this shock was $\approx 130 \mathrm{~km}$. In the downstream region the bulk velocity was about $115 \mathrm{~km} \cdot \mathrm{s}^{-1}$. It is the average value during the time interval from 14:10:50 UT to 14:18:20 UT. (This time interval corresponds to the time interval $650-1100 \mathrm{~s}$ after 14:00:00 UT). Three components of the bulk plasma velocity averaged over 5 time periods are listed in Table 1 . The absolute value of downstream magnetic field, averaged over the time interval studied, 650-1100 seconds, was $26 n T$, averaged density was $n_{d w}=12 \mathrm{~cm}^{-3}$. The downstream region Alfven velocity can be estimated as $V_{a} \approx 164 \mathrm{~km} \cdot \mathrm{s}^{-1}$. The downstream $\beta$ was high: $\beta=8$.

The average magnetic field during the time period $650-1100 \mathrm{~s}$ was $\boldsymbol{B}_{U K S}^{a}=-4.3 ; 14.3 ;-21.8 \mathrm{nT}$ according to UKS data. The angle between $\boldsymbol{B}_{U K S}^{a}$ and the average magnetic field calculated according to IRM data $\boldsymbol{B}_{I R M}^{a}$ was $4.8^{\circ}$. The amplitude difference between amplitudes of $\left|\boldsymbol{B}_{U K S}^{a}\right|$ and $\left|\boldsymbol{B}_{I R M}^{a}\right|$ was smaller then $5 \%$. The $\boldsymbol{B}_{U K S}^{a}$ magnetic field was used below as average for downstream magnetic field.

The bow shock was crossed first by AMPTE UKS and then by AMPTE IRM. The separation vector between the two satellites during the crossing was $\boldsymbol{R}=-50.5 \mathrm{~km} ;-24.6 \mathrm{~km} ; 18.2 \mathrm{~km}$ (GSE).

\section{Downstream waves}

\subsection{Wave spectrum}

The frequency time spectrogram for the shock crossing considered, obtained by spectral analysis of the AMPTE 

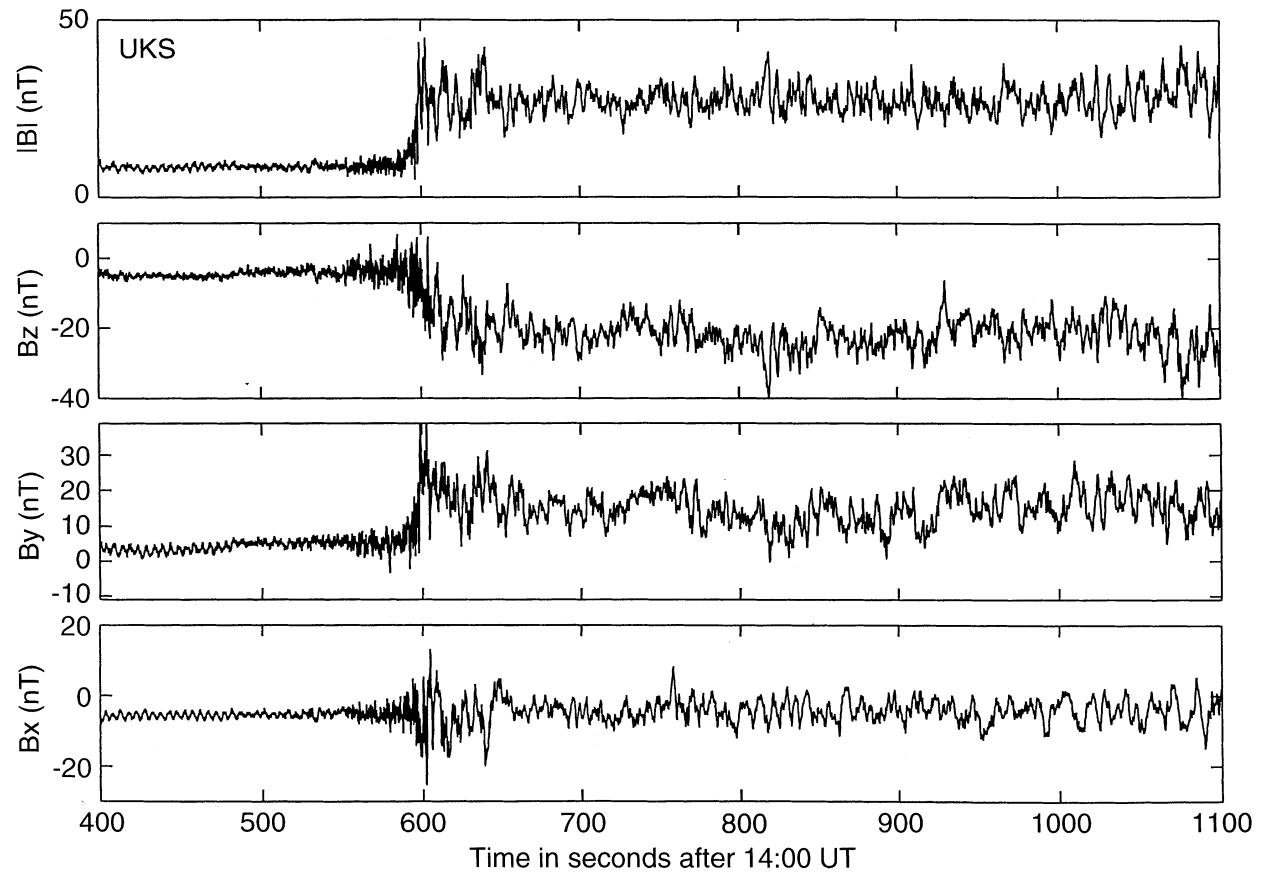

Fig. 1. Absolute value and three components of magnetic field measured by AMPTE UKS during bow shock crossing which occurred at about 14:10:00 UT on 20.10.1984. Time scale is in seconds after 14:00:00. On the figure the moment of the ramp crossing approximately corresponds to $600 \mathrm{~s}$
IRM $\mathrm{B}_{y}$ component of the magnetic field, is shown in Fig. 3. The features of the spectra dynamics are usual for the quasiperpendicular shocks. In the foot the main part of the wave energy resides in the frequency range from about one to a few $\mathrm{Hz}$. The waves in this frequency range are whistlers it was shown by Fairfield (1974). As the satellite approaches the downstream region the wave frequency drops. The main energy of the turbulence is present in the downstream region in the frequency range $0.0-1.0 \mathrm{~Hz}$ as can be seen in Fig. 3. These are the waves that are studied in the present study. The stationarity of the downstream turbulence was studied by the method based on the calculation of the double Fourier transform (e.g., Bendat and Piersol, 1986). The result of that study was that turbulence is stationary for the chosen time interval.

\subsection{The direction of propagation of the downstream waves}

To determine the directions of the wave vector $\boldsymbol{k}$ the standard procedure for calculating the variation matrix (Sonnerup and Cahill 1967) was used. This procedure was applied to the three frequency ranges: $0.1 \mathrm{~Hz}-$ $0.3 \mathrm{~Hz}, 0.3 \mathrm{~Hz}-0.5 \mathrm{~Hz}, 0.5 \mathrm{~Hz}-0.8 \mathrm{~Hz}$. The variation matrix was calculated for five time periods: 14:10:5014:18:20 UT (650-1100 s), 14:10:50-14:12:30 UT (650 750 s), 14:12:30-14:14:10 UT (750-850 s), 14:14:1014:15:50 UT (850-950 s), 14:14:10-14:18:20 UT (850$1100 \mathrm{~s})$. This subdivision was made just to check the deviations of final results with the change of studied time interval. The results of these calculations are presented in Table 2.

Table 2 shows that the smallest eigenvalue can easily be defined for all frequency ranges and time intervals. The direction of the propagation in this case is determined by the direction of the eigenvector, corresponding to this eigenvalue. The eigenvectors corresponding to the smallest eigenvalues are also listed in Table 2. The direction of propagation for almost all the waves studied are close to the $x$-direction. Only the waves in the frequency range $0.1-0.3 \mathrm{~Hz}$ during the period 14:12:30-14:14:10 and in the frequency range $0.3-0.5 \mathrm{~Hz}$ during the period 14:10:50-14:12:30 have comparatively larger angles with the x-axis: 41 and 37 degrees respectively.

For some cases the ratio of the medium eigenvalue to the smallest one is not high enough to give confidence in the direction of $\boldsymbol{k}$ (e.g. $0.5 \mathrm{~Hz}-0.8 \mathrm{~Hz}$ for time interval $14: 10: 50-14: 12: 30$ UT). But the fact that the majority of the smallest eigenvalue vectors (particularly with high ratios of eigenvalues as in the $0.5 \mathrm{~Hz}-0.8 \mathrm{~Hz}$ frequency range for the time interval 14:14:10-14:15:50 UT), have approximately the same direction, gives confidence in the results of the minimum variance analysis.

The minimum variance procedure was also applied to the whole frequency range $0.1-0.8 \mathrm{~Hz}$ for the time period 650-1100 s. Using data from AMPTE-IRM the ratio of the eigenvalues was determined to be $1: 2.3: 2.8$ and the wave vector corresponding to the smallest eigennumber was $\boldsymbol{k}_{I R M} \approx 0.90 ; 0.13 ;-0.41$. From the

Table 1. Components of plasma bulk velocity

\begin{tabular}{lllcl}
\hline $\begin{array}{l}\text { Time } \\
\text { interval }\end{array}$ & $V_{x} \mathrm{~km} \cdot \mathrm{s}^{-1}$ & $V_{y} \mathrm{~km} \cdot \mathrm{s}^{-1}$ & $V_{z} \mathrm{~km} \cdot \mathrm{s}^{-1}$ & $|V| \mathrm{km} \cdot \mathrm{s}^{-1}$ \\
\hline $650-750$ & -100 & 21 & -10 & 103 \\
$750-850$ & -117 & 17 & 18 & 120 \\
$850-950$ & -117 & 33 & 9 & 122 \\
$950-1100$ & -114 & 35 & 10 & 120 \\
$850-1100$ & -115 & 34 & 10 & 120 \\
$650-1100$ & -111 & 27 & 8 & 115 \\
\hline
\end{tabular}



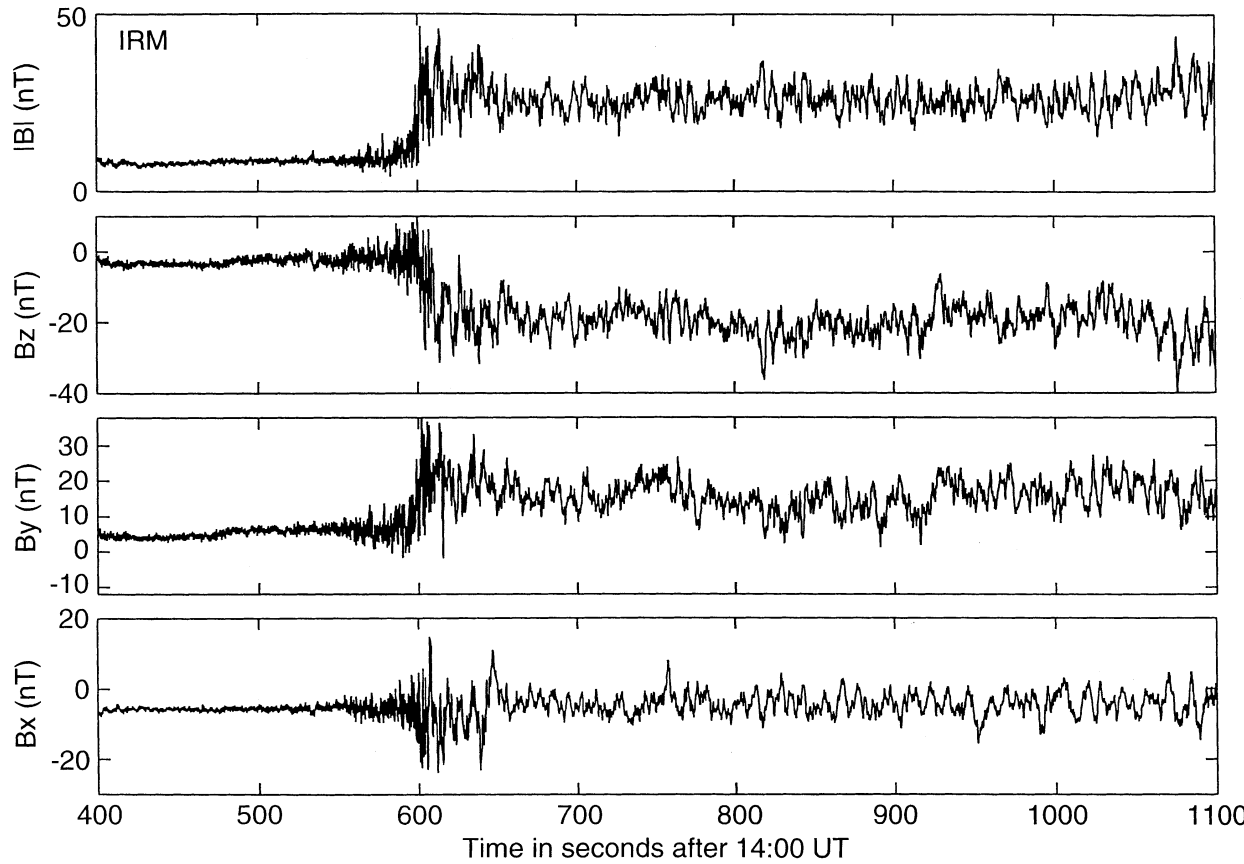

Fig. 2. Absolute value and three components of magnetic field measured by AMPTE IRM during bow shock crossing which occurred at about 14:10:00 UT on 20.10.1984. Time scale is in seconds after 14:00:00

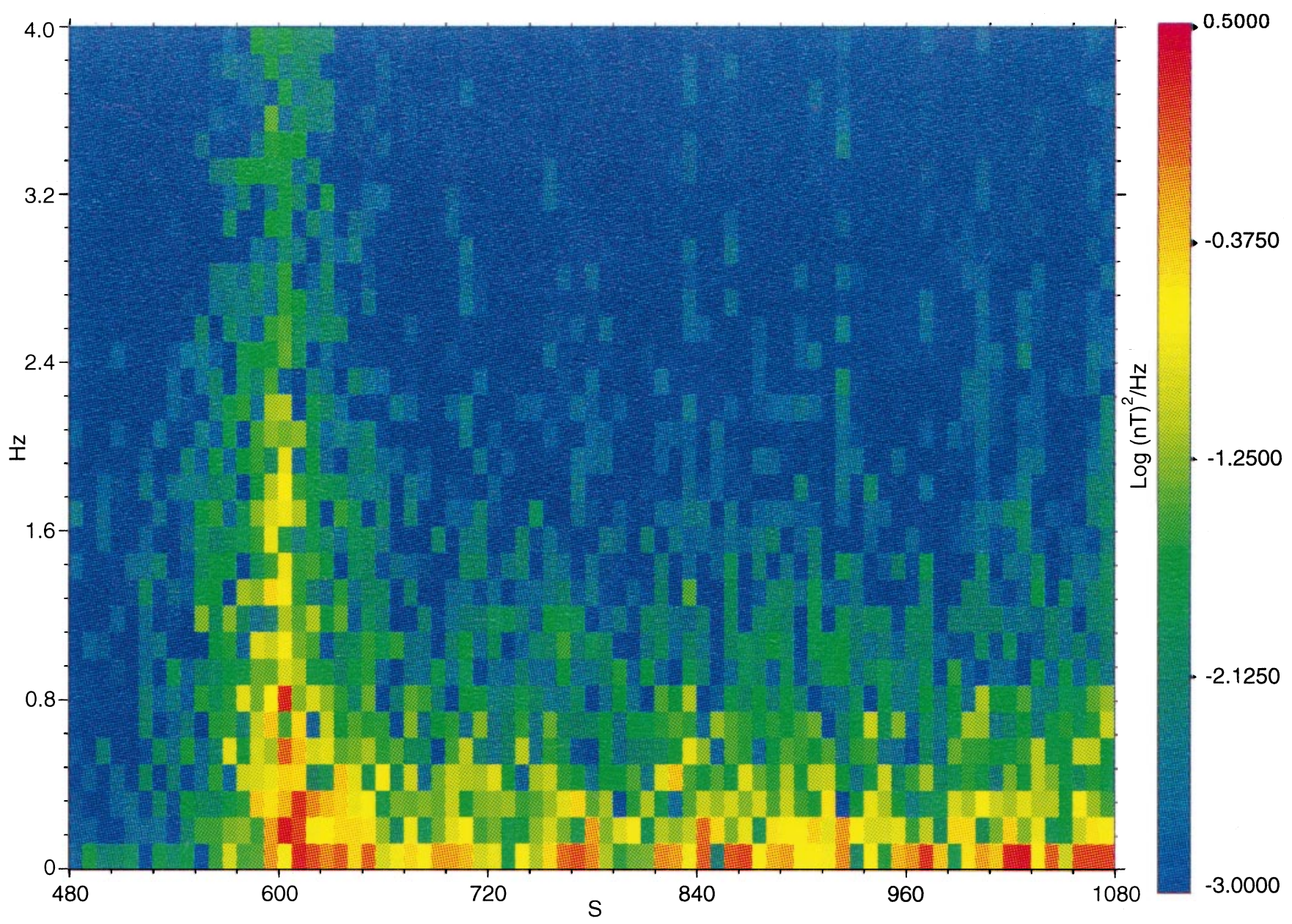

Fig. 3. Dynamic spectrum of $B_{y}$ component of magnetic field measured by AMPTE IRM during bow shock crossing at about 14:10:00 UT on 20.10.1984. Time scale is in seconds after 14:00:00 UT. 
Table 2. The ratio of three eigenvalues of variational matrix calculated for three frequency ranges during five time periods and the wave vector corresponding to the smallest eigennumber

\begin{tabular}{llll}
\hline Time & $0.1-0.3 \mathrm{~Hz}$ & $0.3-0.5 \mathrm{~Hz}$ & $0.5-0.8 \mathrm{~Hz}$ \\
$14: 00$ & & & \\
\hline $650-$ & $1.0: 1.8: 2.4$ & $1.0: 1.8: 3.0$ & $1.0: 1.9: 3.5$ \\
-750 & $(0.96,0.12,-0.24)$ & $(0.80,0.57,-0.20)$ & $(0.98,0.15,-0.10)$ \\
$750-$ & $1.0: 2.7: 5.9$ & $1.0: 2.5: 3.2$ & $1.0: 2.8: 3.3$ \\
-850 & $(0.76,0.44,-0.47)$ & $(0.95,0.27,-0.15)$ & $(0.99,0.05,-0.08)$ \\
$850-$ & $1.0: 2.1: 2.6$ & $1.0: 3.3: 4.1$ & $1.0: 4.7: 5.8$ \\
-950 & $(0.94,0.21,-0.28)$ & $(0.98,-0.06,0.20)$ & $(-0.97,0.22,0.07)$ \\
$850-$ & $1.0: 1.8: 2.3$ & $1.0: 2.3: 2.9$ & $1.0: 3.0: 3.5$ \\
-1100 & $(0.97,0.13,-0.23)$ & $(0.99,-0.04,0.04)$ & $(-0.99,0.01,0.06)$ \\
$650-$ & $1.0: 2.1: 2.7$ & $1.0: 2.1: 2.4$ & $1.0: 2.9: 3.2$ \\
-1100 & $(0.91,0.23,-0.35)$ & $(0.99,0.15,-0.04)$ & $(0.99,0.02,-0.07)$ \\
\hline
\end{tabular}

UKS data the eigenvalue ratios were $1: 2.3: 2.6$ and the wave vector $\boldsymbol{k}_{U K S} \approx 0.90 ; 0.17 ;-0.41$. This shows that the application of the minimum variance procedure gives approximately the same results for both satellites.,

\subsection{Cospectral characteristics of downstream waves}

The coherency function and the phase were calculated on the basis of Eqs. $(1,2)$. The coherency function and the phase calculated for the z-components of the magnetic field measured by AMPTE UKS and AMPTE IRM at 14:10:50-14:18:20 UT are shown in Figs. 4, 5. The magnitude of the coherency function is high enough in the low frequency region and drops with increasing frequency. Some increase of the coherency function which can be seen above $3.0 \mathrm{~Hz}$ is artificial. This increase is connected with small amplitudes of waves in this frequency range. It appears because both the numerator and denominator in Eq. (1) are small in this case. The variation of the phase can be used to reach conclusions about the real level of coherency in that frequency range. More information can be seen in the phase function. In the frequency range $0.02-1.0 \mathrm{~Hz}$ approximately the linear trend of the phase can be easily seen. The variation of the calculated values of the phase increases with increasing frequency. Instead of a wellrecognized linear trend above $1 \mathrm{~Hz}$ the calculated values of the phase are spread over a wider range, with a much greater variation. The comparison of the variation of the phase below and above $1 \mathrm{~Hz}$ shows the relatively high level of the true coherency value below $1 \mathrm{~Hz}$. The slope of the linear trend below $1 \mathrm{~Hz}$ is:

$\alpha=\frac{\Delta_{\text {phase }}(\mathrm{rad})}{\Delta \omega_{1}}=0.75 \mathrm{~s}$,

where $\omega_{1}$ is the observed frequency. This corresponds to the time shift of $0.75 \mathrm{~s}$. according to Eq. (3). The phase velocity of waves in the frequency range in which the linear trend is present can be estimated as $62.5 \mathrm{~km} \cdot \mathrm{s}^{-1}$ and is directed downstream in the satellite reference

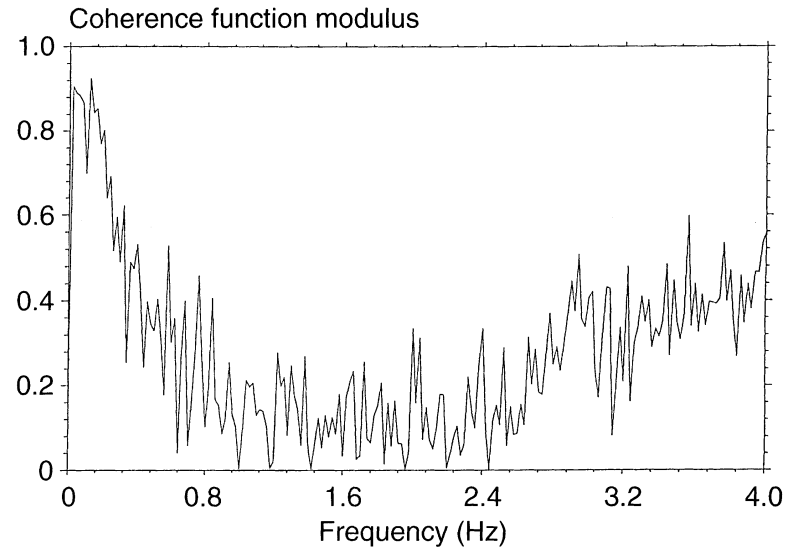

Fig. 4. Modulus of coherency function calculated for $B_{z}$ components of AMPTE UKS and AMPTE IRM magnetic fields for the time interval $650-1100 \mathrm{~s}$

frame. In the plasma frame the waves propagate in the upstream direction but they are convected downstream by the plasma flow.

\subsection{Determination of $K$ vector, plasma frame frequency and the dispersion relation}

To determine the dependence of the wave vector $\boldsymbol{k}$ upon the observed frequency $\omega_{1}$ we need at first to put the linear function $\alpha \omega_{1}$ instead of $\Psi\left(\omega_{1}\right)$ into Eq. (6) and rewrite it as:

$|k|\left(\omega_{1}\right)[\mathrm{km}]^{-1}=\frac{\omega_{1} 0.75[\mathrm{sec} .]+\omega_{1} 0.035[\mathrm{sec} .]}{|59[\mathrm{~km}]| \cos (\vartheta)+V_{0} 0.035[\mathrm{sec} .] \cos (\varphi)}$

As was shown, directions of propagation, plasma bulk velocity and satellite separation vector are close to the $\mathrm{x}$-axis direction. The $\cos (\vartheta)$ and $\cos (\varphi)$ have the same order of magnitude. The absolute value of the plasma bulk velocity is about $115 \mathrm{~km} \cdot \mathrm{s}^{-1}$ so it is possible to disregard the second term in the denominator of Eq. (8)

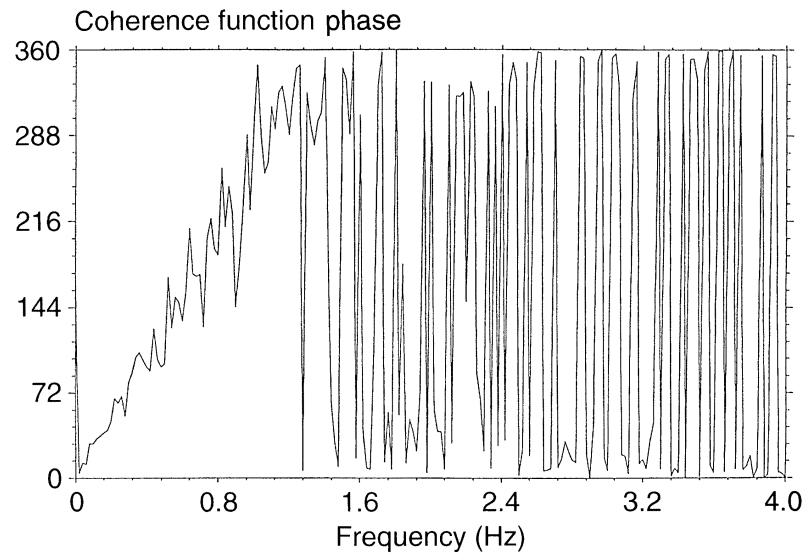

Fig. 5. Phase function calculated for $B_{z}$ components of AMPTE UKS and AMPTE IRM magnetic fields for the time interval 650 $1100 \mathrm{~s}$. The units for $y$-axis of this plot are degrees 
in comparison with its first term. Instead of Eq. (7) we can use the simplified equation:

$|k|\left(\omega_{1}\right)=\frac{\Psi\left(\omega_{1}\right)}{|R| \cos (\vartheta)}$

The resulting dependence of the wave vector upon observed frequency is shown in Fig. 6. We calculate this dependence only in the region of observed frequency where a well-defined trend is seen in the phase function i.e., in the frequency range $0.02-1.0 \mathrm{~Hz}(\approx 0.125$ $\left.6.28 \mathrm{rad} \cdot \mathrm{s}^{-1}\right)$. The dependence of the wavelength upon observed frequency is shown in Fig. 7. It follows from this figure that the observed waves have wavelengths in the range $\approx 10^{2}-10^{3} \mathrm{~km}$.

The next step is to obtain the correspondence between the observed frequency $\omega_{1}$ and the frequency in the plasma reference frame $\omega_{p . f}$. on the basis of Eq. (5). However, first we should note that the wave vectors for all case are directed upstream, so the second term on the right hand side of Eq. (6) is negative. The direction of wave propagation in the plasma rest frame is opposite to the direction of waves in the satellite frame, so the signs of $\omega_{1}$ and $\omega_{p . f}$. must be opposite. (This corresponds to the change of the direction of polarization.) Thus, Eq. (6) can be rewritten for the case considered as:

$\left|\omega_{p . f .}\right|=-\left|\omega_{1}\right|+\left|\boldsymbol{k} \boldsymbol{V}_{0}\right|$.

We use the value of the angle between wave vector and plasma wave velocity averaged over the full time interval $14: 10: 50-14: 18: 20$, separately for three observed frequency intervals: $0.1-0.3 \mathrm{~Hz}, 0.3-0.5 \mathrm{~Hz}$ and $0.5-1 \mathrm{~Hz}$. The plasma frame frequency range of the observed waves continues far above the ion cyclotron frequency. Finally the dispersion relation between $\omega_{p . f}$. and $|k|$ obtained from Eqs. $(8,9)$ is shown in Fig. 8. It can be seen from this that the dispersion relation is linear in the frequency range considered. The linear slope of this dispersion was obvious from the linear dependence of phase angle upon observed frequency.

\section{Discussion}

The uniform continuous dependence of the phase upon the observed frequency in the range $0-1.0 \mathrm{~Hz}$ is a sign that waves observed in these frequencies have the same mode. As can be seen from the dynamic spectrogram, the main energy of downstream wave turbulence stored is also below $1 \mathrm{~Hz}$. The identification of the mode of these wave is the identification of the most developed mode in the downstream region. To determine which mode we can attribute the observed waves to we need to compare the dispersion relations and propagation properties of possible candidates. In addition to three MHD modes the mirror mode, and Alfvenic ion cyclotron waves are usually observed downstream of a quasiperpendicular shock front (Lacombe et al., 1990; 1992; Winske and Quest, 1988). The last two modes are unstable due to the anisotropy in the ion temperature

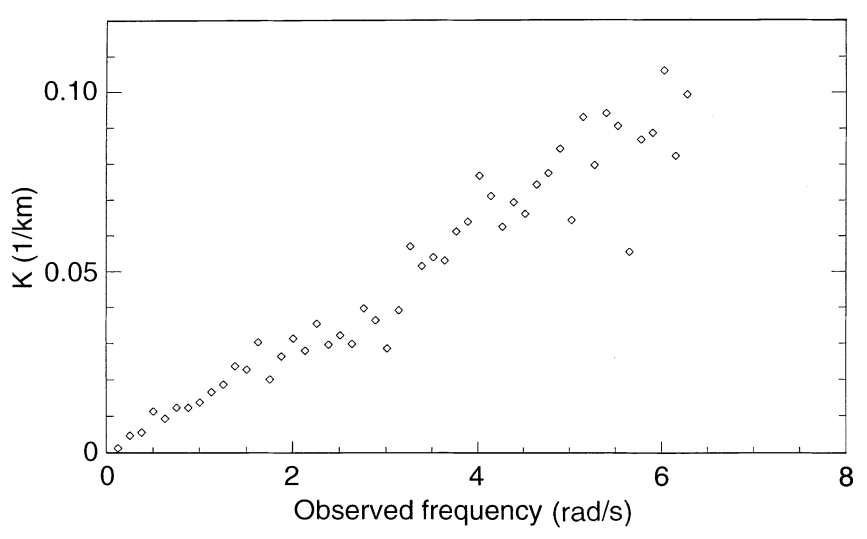

Fig. 6. Dependence of wave vector upon observed frequency

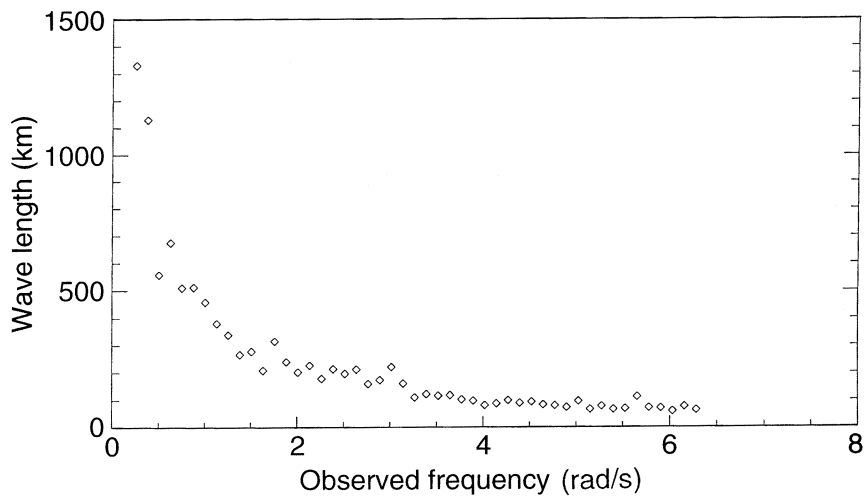

Fig. 7. Dependence of wave length upon observed frequency

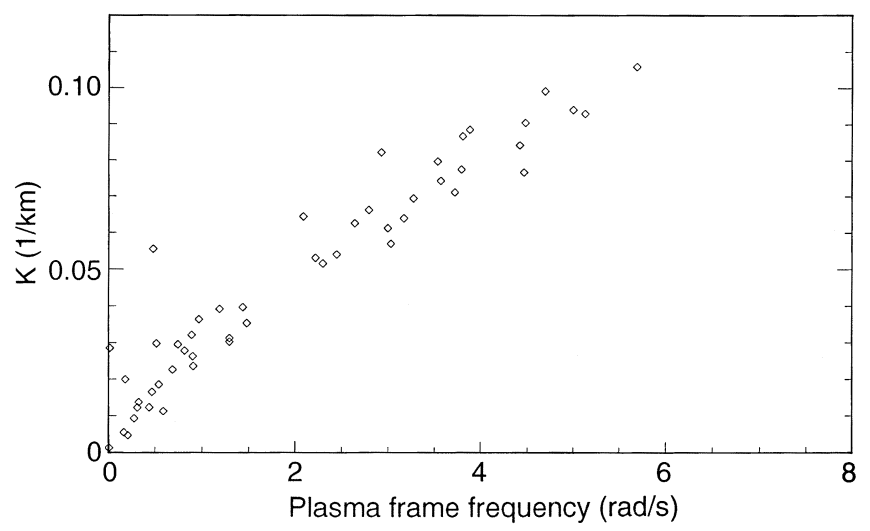

Fig. 8. Dependence of wave vector upon plasma frame frequency

which is present downstream from the shock front. The mirror mode can be excluded from consideration because it has no velocity in the plasma rest frame. The largest growth rate of Alfven ion cyclotron waves is for propagation parallel to the magnetic field. In our case, as can be seen from Table 2, waves are propagating almost perpendicular to the magnetic field. This fact together with the existence of waves above the ion cyclotron frequency, also helps to exclude Alfven ion cyclotron waves. 
Three MHD modes; fast magnetosonic, intermediate (Alfvenic) and slow magnetosonic, were comprehensively studied analytically (e.g., Akhiezer et al., 1975; Formisano and Kennel, 1969) and their propagation properties are well known in a wide range of plasma parameters. The dispersion relation for all three wave modes from Formisano and Kennell, (1969) are shown in Fig. $9\left(C_{s}>V_{a}\right.$ is the case which corresponds to the high $\beta$ situation). As can be seen from these curves, well below the lower resonance frequency $\Omega_{r_{1}}=\Omega_{+} \cos (\theta)$ all three dispersion relations are linear. The phase velocity of the slow wave decreases as the frequency approaches $\Omega_{r_{1}}$ (where $\Omega_{+}$is the ion cyclotron frequency and $\theta$ is the angle between wave vector and magnetic field direction). Above $\Omega_{r_{1}}$ only two branches exist; fast magnetosonic and intermediate. The ion gyrofrequency $\Omega_{+}$downstream of the considered shock is about $2.45 \mathrm{rad} \cdot \mathrm{s}^{-1}=2 \pi 0.78 \mathrm{~Hz}$, the angle between the direction of the wave propagation and the downstream magnetic field exceeds $70^{\circ}$, so the resonance frequency in our case is $\Omega_{r_{1}}<0.85 \mathrm{rad} \cdot \mathrm{s}^{-1}=2 \pi$ $0.27 \mathrm{~Hz}$. A substantial portion of the observed waves have plasma rest frequencies higher than this value. So the waves in the observed frequency range $0.02-1.1 \mathrm{~Hz}$ cannot be attributed to the slow magnetosonic branch because, as mentioned, they must have the same type in the whole observed frequency range $0.1-0.8 \mathrm{~Hz}$. Also a deviation from the linear dispersion must be seen for the slow magnetosonic branch in the vicinity of the resonance frequency, $\Omega_{r_{1}}$, whereas the observed waves have a well-recognized linear dispersion in that frequency range.

To choose between fast magnetosonic and intermediate branches we need to compare their phase velocity with the phase velocity of the observed waves. According to Akhiezer et al., (1975) the phase velocity of fast magnetosonic and intermediate waves are determined by the following equations:

$V_{\text {fast }}=\sqrt{\frac{V_{A}^{2}+C_{s}^{2}+\sqrt{\left(V_{A}^{2}+C_{s}^{2}\right)^{2}-4 V_{A}^{2} C_{s}^{2} \cos ^{2}(\theta)}}{2}}$.

$V_{\text {int }}=V_{a} \cos (\theta)$

The phase velocity of the fast magnetosonic wave is higher than the Alfven velocity for the whole range of angles. The value of averaged phase velocity was $V_{\text {ph.av. }} \approx 37.5 \mathrm{~km} \cdot \mathrm{s}^{-1} \approx 0.23 V_{a}$. So $V_{\text {ph.av. }}$ is much smaller than possible for the phase velocity of fast magnetosonic waves. To compare this velocity with the $V_{\text {int }}$ we need to point out that the angle between the downstream magnetic field, the median directions of propagation $\boldsymbol{k}_{m}=\frac{1}{2}\left(\boldsymbol{k}_{U K S}+\boldsymbol{k}_{I R M}\right)$ for this time interval is $74^{\circ}$. The phase velocity of an intermediate wave travelling at such an angle to the ambient magnetic field, must be:

$V_{\text {int }}=V_{a} \cos \left(74^{\circ}\right) \approx=45 \mathrm{~km} \cdot \mathrm{s}^{-1}$

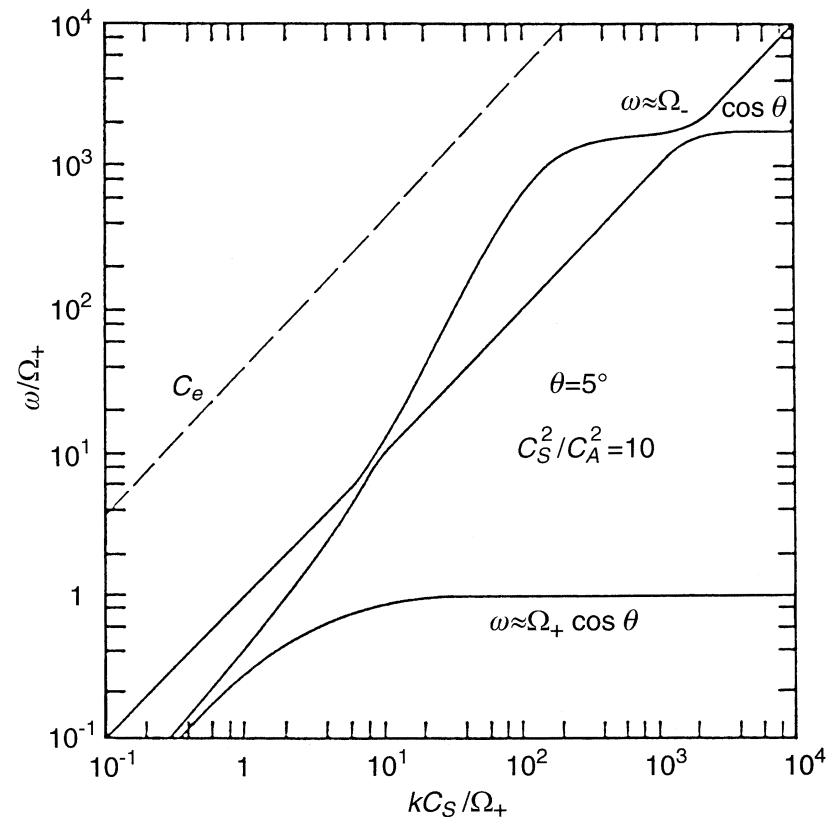

Fig. 9. Dispersion relations for 3 MHD modes from Formisano and Kennel, (1969) calculated for the case $\beta>1$

The discrepancy between $V_{\text {int }}$ and $V_{\text {ph.av. }}$ is small, and can be attributed to a few degrees error in the estimation of the directions of the wave vector and the magnetic field direction. If $\boldsymbol{k}_{m}$ as a direction of the wave propogation is replaced with a direction averaged over the eigenvectors listed in Table 2 , this results in a propagation angle of $\approx 80^{\circ}$ to the magnetic field. This discrepancy does not affect the conclusion that the phase velocity of the observed waves is much closer to the intermedite wave velocity than to the fast mode velocity as determined from a two fluid plasma model. It is worth noting that in Fig. 8 the dispersion relation for experimentally observed waves in the plasma with $\beta>1$ is shown. It might not be suprising that this dispersion relation differs from dispersion relations obtained in the two fluid idealization. The comparison of the obtained dispersion relation with the simulation results in Krauss-Varban et al., (1994), makes little sense because the plasma distribution functions in the downstream region of a supercritical quasiperpendicular shock are not Maxwellian (e.g., Sckopke et al., 1983).

There is a disagreement between the result, obtained in the present paper and previous studies of downstream turbulence (Lacombe et al., 1990, 1992) where mirror waves were observed in a similar frequency range. This disagreement can be attributed to two factors. First mirror waves were not observed in downstream regions of all shocks (Lacombe et al., 1992). Secondly it may be that in the case of high $\beta$ a possible anisotropy of temperature caused by reflected ions plays a different role to that in the case of low $\beta$ shocks. 


\section{Conclusions}

A method for the determination of the mode of an observed plasma wave on the basis of two point magnetic field measurements was presented.

This method was applied to the waves observed by AMPTE UKS and AMPTE IRM downstream of the quasiperpendicular shock on the 20 October 1984. It was established that the plasma turbulence downstream of the shock mainly consists of waves observed below $1.0 \mathrm{~Hz}$. These waves propagate upstream in the plasma rest frame but are convected downstream by the plasma flow in the shock reference frame. The wavelengths of these waves are between about $10^{2}-10^{3} \mathrm{~km}$, observed frequencies are between $0.05-6.0 \mathrm{rad} \cdot \mathrm{s}^{-1} \approx 0.2-2.5 \omega_{+}$.

The velocity of these waves is close to the velocity of intermediate waves propagating at the same angle as determined from a two fluid model of plasma.

Acknowledgements. The authors acknowledge valuable discussions with Dr. S. Walker, Dr. V.V. Krasnosel'skikh, Dr. D. Lagoutte, and Dr. C. Lacombe. Software IMOS, which was developed in LPCE by Dr. Lagoutte and P. Latremoliere, was used in the data analysis. AMPTE IRM data were kindly provided by Dr. H. Lühr and Dr. W. Baumjohann. AMPTE UKS data were kindly provided by Prof. D. Southwood (magnetic field), Prof. S.J. Schwartz and Dr. A. Coates (plasma data). M. Balikhin was funded by a grant from the UK PPARC during the preparation of the final version of the work.

Topical Editor K.-H. Glaßmeier thanks U. Motschmann and another referee for their help in evaluating this paper.

\section{References}

Akhiezer, A. I, I. A. Akhiezer, R. V. Polovin, A. G. Sitenko, and K. N. Stepanov, Plasma electrodynamics, vol 1: linear theory, Pergamon, 1975.

Balikhin M. A., and M. A. Gedalin, The comparative analysis of different methods for distinguishing of temporal and spatial variations, in Spatial-temporal analysis for resolving plasma turbulence (START), ESA WPP-047, 183-186, 1993.

Beall J. M, Y. C. Kim, and E. J. Powers, Estimation of wavenumber and frequency spectra using fixed probe pairs, J. Appl. Phys., 53(6), 3933-3940, 1982.

Bendat J. S., and A. G. Piersol, Random Data, John Wiley, 1986.

Coates A. J., J. A. Bowles, R. A. Gowen, B. K. Hancock, A. D. Jonstone, and S. J. Kellock, The AMPTE UKS threedimensional ion experiment, Geosci., Remote Sensing, GE-23, 287-292, 1985.

Dudok de Wit T., V. V. Krasnosel'skikh, S. D. Bale, M. W., Dunlop, H. Lühr, S. J. Schwartz and L. J. C. Woolliscroft, Determina- tion of dispersion relations in quasi-stationary plasma turbulence using dual satellite data, Geophys. Res. Lett., 22, 26532656, 1995.

Fairfield D. H., Whistler waves observed upstream from collissionless shock, J. Geophys. Res., 79, 1368-1378, 1974.

Formisano, V., and C. F. Kennel, Small amplitude waves in high $\beta$ plasmas, J. Plasma Phys., 3, 55-74 1969.

Grenander U., and M. Rosenblatt, Statistical analysis of stationary time series, John Wiley, New York, Almqvist and Wiksell, Stockholm, 1957.

Krauss-Varban D., N. Omidi, and K. B. Quest, Mode properties of low frequency waves: kinetic theory versus Hall-MHD, J. Geophys. Res., 99, 2958-6009, 1994.

Lacombe C., E. Kinzelin, C. C. Harvey, D. Hubert, A. Mangeney, J. Elaoufir, D. Burgess, and C.T. Russell, Nature of the turbulence observed by ISEE-1,2 during a quasi-perpendicular crossing of the Earth's bow shock, Ann. Geophysicae, 8, 489$502,1990$.

Lacombe C., F. G. E. Pantellini, D. Hubert, C. C. Harvey, A. Mangeney, G. Bemont, and C. T. Russell, Mirror and Alfvenic waves observed by ISEE-1,2 during crossings of the Earth's bow shock, Annales Geophysicae, 10, 772-784, 1992.

Lühr H., N. Klöcker, W. Oelschlägel, B. Häusler, and M. Acuna, The IRM fluxgate magnetometer, Geosci., Remote Sensing, GE23, 259-261, 1985.

Otnes R. K., and L. Enochson, Applied time series analysis, vol, John Wiley, New York, 1978.

Rodriguez, P., and D. Gurnett, Electrostatic and electromagnetic turbulence associated with the Earth's bow shock, J. Geophys. Res., 80, 19-31, 1975.

Schwartz S. J., D. Burges, W. Wilkinson, R. L. Kessel, M. Dunlop, and H. Lühr, Observations of short large-amplitude magnetic structures at a quasi-parallel shock, J. Geophys. Res., 93, 42094227, 1992.

Sckopke, N., G. Paschmann, S. J. Bame, J. T. Gosling, and C. T. Russell, Evolution of ion distributions across the nearly perpendicular bow shock: specularly and non-specularly reflected-gyrating ions, J. Geophys. Res., 88, 6121-6136, 1983.

Sonnerup B. U. O., and L. Y. Jr. Cahill, Magnetopause structure and altitude from Explorer-12 observations, J. Geophys. Res., 72, 171-183, 1967.

Southwood D. J., W. A. C. Mier-Jedrzejowicz and C. T. Russell, The fluxgate magnetometer for AMPTE UKS subsatellite, Geosci., Remote Sensing, GE-23, 301-304, 1985.

Vaisberg O. L., A. A. Galeev, G. N. Zastenker, S. I. Klimov, M. N. Nozdrachev, R. Z. Sagdeev, A. Yu. Sokolov, and V. D. Shapiro, Electron acceleration in the front of collisionless shock waves, Rep 813, USSR, Acad. Sci., Space Res. Inst., Moscow, 1983.

Winske D., and K. B. Quest, Magnetic field and density fluctuations at perpendicular supercritical collisionless shock, J. Geophys. Res., 93, 9681-9693, 1988. 